\title{
LEVANTAMENTO QUANTITATIVO DA ARBORIZAÇÃO DE PRAÇAS DA CIDADE DE CÁCERES/MT
}

Katiúscia Christini de Assunção ${ }^{1}$; Petterson Baptista da Luz ${ }^{2}$; Leonarda Grillo Neves ${ }^{3}$; Severino de Paiva Sobrinho ${ }^{34}$

\section{RESUMO}

O estudo teve objetivo de realizar um levantamento quantitativo das árvores e palmeiras existentes em quatro praças na área central de Cáceres/MT. Foram estudadas quatro praças: José de Anchieta, Barão do Rio Branco, Duque de Caxias e Benjamim Constant. Com auxilio de literatura especifica e profissionais da área foram feita a identificação das espécies. Identificaram-se 231 árvores e palmeiras distribuídas nas quatro praças amostradas. Foram identificadas 12 famílias, 22 gêneros e 25 espécies. A Praça José de Anchieta possui o maior número de árvores e palmeiras (68). Observou-se presença de cupins e erva-de-passarinho em 17,64\%. A espécie Licania tomentosa estava presente em todas as praças, com total de 42 exemplares (18,2\%). A família com maior freqüência foi Arecaceae, com 65 indivíduos (28,2\%). Constatou-se que 135 (58,44\%) dos indivíduos são nativos e 96 (41,56\%) exóticos. O conhecimento sobre as espécies é de fundamental importância para conservação e manutenção do espaço público, viabilizando ou melhorando a qualidade desses espaços, oferecendo conforto e lazer as pessoas que os freqüentam.

Palavras- chave: paisagismo; árvores; Arecaceae; espaço público.

\section{QUANTITATIVE SURVEY OF ARBORIZATION OF SQUARES IN CÁCERES / MT, BRAZIL}

\section{ABSTRACT}

The study had the objective to achieve a quantitative survey of the trees and palms existing into four squares on the central area of Cáceres, MT. We studied four squares: José de Anchieta, Barão do Rio Branco, Duque de Caxias and Benjamin Constant. With the aid of specific literature and professionals we identified 231 trees and palms distributed in the four squares sampled. We identified 12 families, 22 genera and 25 species. The Square José de Anchieta has the largest number of trees and palms (68). We observed the presence of termites and herb-of-finch (Struthanthus flexicaulis) in $17.64 \%$. The species Licania tomentosa was present in all the squares with 42 specimens (18.2\%). The most frequent family was Arecaceae with 65 individuals (28.2\%). It was found that 135 (58.44\%) of individuals are native and 96 (41.56\%) exotic. Knowledge on species is of fundamental importance for the preservation and maintenance of the public space, making or improving the quality of these spaces, providing comfort and leisure for people who frequent them.

Keywords: landscaping; trees; Arecaceae; public area.

1 Acadêmica de Agronomia da Universidade do Estado de Mato Grosso UNEMAT, Campus Universitário de Cáceres, 78200-000, Cáceres-MT. E-mail: katiusciauxa@hotmail.com

2 Professor Doutor. Departamento de Agronomia, Universidade do Estado do Mato Grosso/UNEMAT, Campus Universitário de Cáceres, 78200-000, Cáceres-MT. E-mail: petterbaptista@yahoo.com.br.

3 Professor (a), Departamento de Agronomia, Universidade do Estado do Mato Grosso/UNEMAT, Campus Universitário de Cáceres, 78200-000, Cáceres-MT. E-mail: leonardaneves@unemat.br, paivasevero@unemat.br

${ }^{4}$ recebido em 23.07.2012 e aceito para publicação em 15.03.2014 


\section{INTRODUÇÃo}

A vegetação é importante por oferecer vantagens às pessoas, tais como: bem-estar psicológico; melhor efeito estético; sombra para os pedestres e veículos; proteção contra o vento e seu direcionamento; amortecimento do som; amenização da poluição sonora; redução do impacto da água de chuva e seu escorrimento superficial; auxílio na diminuição da temperatura, pois absorvem os raios solares e refrescam o ambiente pela grande quantidade de água transpirada pelas folhas; melhoria da qualidade do ar, e preservação da fauna silvestre (PIVETTA \& SILVA FILHO, 2002).

A paisagem urbana vem sofrendo alterações, decorrentes do aumento da população urbana, com presença de casas, indústrias, sistema viário, estrutura e equipamentos das empresas de energia elétrica, água, saneamento e telecomunicação (CEMIG, 2001).

Assim, o planejamento paisagístico é fundamental para a conservação de árvores, que são componentes de grande importância para a qualidade de vida da população.

A arborização urbana, no Brasil, é considerada um tema recente, de evolução lenta e no qual as administrações públicas e a comunidade devem se envolver, cumprindo papéis distintos. Atualmente, em cidades onde ocorre o planejamento das arborizações, a preocupação é tornar o ambiente urbano diversificado quanto às espécies empregadas, mais homogêneo e envolvente com a paisagem circundante (MELO \& ROMANINI, 2008).

De maneira geral, os parques urbanos mostram-se como unidades urbanísticas fundamentais para a vida nas cidades. Além da elevada área física, a cobertura vegetal é uma característica marcante nestes espaços, sendo um dos componentes de grande importância na formação da paisagem local (SILVA et al., 2007a).

Para conhecer a arborização da cidade, é necessário fazer um levantamento para avaliar a situação e, por meio dos resultados obtidos, será possível verificar as soluções possíveis para cada local.

Esse estudo, na cidade de Cáceres, faz-se necessário por ser uma cidade antiga sem planejamento paisagístico, e para orientar o manejo das árvores e palmeiras já existentes e dar subsídios para futuras intervenções e melhoria desses locais.

Portanto, o presente trabalho tem como objetivo realizar um levantamento quantitativo das árvores e palmeiras existentes em quatro praças na área central de Cáceres/MT de modo a fornecer subsídios para o planejamento e a aplicação de medidas mitigadoras.

\section{MATERIAL E MÉTODOS}

\section{Área de estudo}

O estudo foi realizado no município de Cáceres, com as coordenadas $16^{\circ} 04^{\prime} 16^{\prime \prime}$ 'S e $57^{\circ} 40^{\prime} 44^{\prime \prime}$ O, localizado no interior do Estado de Mato Grosso, a $211 \mathrm{~km}$ da capital Cuiabá, com área de aproximadamente 24.398,399 $\mathrm{km}^{2}$ e população estimada de 87.261 habitantes, segundo IBGE (2009).
Os locais visitados no município foram: Praça Barão do Rio Branco, Praça Duque de Caxias; Praça Benjamim Constant, conhecida como Praça da Cavalhada, e Praça José de Anchieta, conhecida como Praça da PM, todas na região central da cidade. 


\section{Procedimentos Metodológicos}

A coleta dos dados foi realizada por meio de visitas aos locais, nos meses de agosto e setembro de 2009.

Os registros das informações foram feitos com auxílio de máquina fotográfica e planilhas.

O modelo da planilha foi igual para todos os locais. Os itens levantados foram os seguintes: nome vulgar, nome cientifico, fitossanidade, sistema radicular (aparente ou não aparente, sendo verificado se a raiz estava afetando calçada ou rua), posição da fiação com relação à copa das árvores, tipo de poda, obstrução aos pedestres.

As espécies foram identificadas com consultas a literatura e a profissionais da área de botânica, os dados foram agrupados em famílias, gêneros, espécies, exóticas e nativas.

Após a coleta dos dados, os mesmos foram processados para a realização da análise descritiva.

\section{RESULTADOS E DISCUSSÃO}

No levantamento, foram identificadas 231 árvores e palmeiras distribuídas nas praças amostradas, representadas em 12 famílias, 22 gêneros e 25 espécies (TABELA 01).

Nas praças estudadas, foram encontradas com predominância as seguintes espécies: oiti (18,2\%),

TABELA 01: Relação das famílias, espécies, origem, número de indivíduos e da freqüência dos exemplares vegetais encontrados em quatros praças da área central na cidade de Cáceres/ MT, 2009. UNEMAT, 2009

\begin{tabular}{|c|c|c|c|c|c|}
\hline Nome vulgar & Família & Nome científico & Origem & $\begin{array}{c}\mathrm{N}^{\circ} \\
\text { indivíduos }\end{array}$ & $\begin{array}{c}\text { Freqüência } \\
\text { (\%) }\end{array}$ \\
\hline Bocaiúva & Arecaceae & $\begin{array}{l}\text { Acrocomia aculeata } \\
\text { (Jacq.) Lodd }\end{array}$ & $\mathrm{N}$ & 19 & 8,2 \\
\hline Cajueiro & Anacardiaceae & $\begin{array}{c}\text { Anacardium occidentale Franz } \\
\text { Eugen Köhler }\end{array}$ & $\mathrm{N}$ & 1 & 0,4 \\
\hline Cambará & Vochysiaceae & Vochysia divergens Pohl & $\mathrm{N}$ & 26 & 11,3 \\
\hline $\begin{array}{l}\text { Canafístula } \\
\text { faveiro }\end{array}$ & $\begin{array}{c}\text { Fabaceae - } \\
\text { Caeasalpinioideae }\end{array}$ & $\begin{array}{c}\text { Peltophorum dubium } \\
\text { (Sprengel) Taubert }\end{array}$ & $\mathrm{N}$ & 9 & 3,9 \\
\hline Chuva de ouro & $\begin{array}{c}\text { Fabaceae - } \\
\text { Caeasalpinioideae }\end{array}$ & Cassia fistula & $\mathrm{E}$ & 2 & 0,9 \\
\hline Cumbaru & $\begin{array}{c}\text { Fabaceae- } \\
\text { Papilonoidae }\end{array}$ & Dipteryx alata Vogel & $\mathrm{N}$ & 1 & 0,4 \\
\hline
\end{tabular}




\begin{tabular}{|c|c|c|c|c|c|}
\hline $\begin{array}{l}\text { Figueira } \\
\text { benjamina }\end{array}$ & Moraceae & Fícus benjamina L. & $E$ & 11 & 4,8 \\
\hline Figueira lira & Moraceae & Fícus lyrata Warb & $\mathrm{E}$ & 8 & 3,5 \\
\hline $\begin{array}{l}\text { Figueira } \\
\text { branca }\end{array}$ & Moraceae & $\begin{array}{c}\text { Ficus gomelleira } \\
\text { Kth. et Bouché }\end{array}$ & $\mathrm{E}$ & 2 & 0,9 \\
\hline Flamboyant & $\begin{array}{c}\text { Fabaceae- } \\
\text { Caeasalpinioideae }\end{array}$ & $\begin{array}{c}\text { Delonix regia } \\
\text { (Hook.) Raf }\end{array}$ & $\mathrm{E}$ & 17 & 7,4 \\
\hline Ipê & Bignoniaceae & Tabebuia sp. & $\mathrm{N}$ & 21 & 9,1 \\
\hline Ipê mirim & Bignoniaceae & $\begin{array}{l}\text { Tecoma stans } \\
\text { (L.) Juss. ex Kunth }\end{array}$ & $E$ & 2 & 0,9 \\
\hline Mangueira & Anacardiaceae & Mangifera indica L. & E & 5 & 2,2 \\
\hline Mogno & Meliaceae & $\begin{array}{c}\text { Swetenia macrophyla } \\
\text { R.A.King }\end{array}$ & $\mathrm{N}$ & 1 & 0,4 \\
\hline Monguba & Bombacaceae & Pachira aquática Aubl. & $\mathrm{N}$ & 1 & 0,4 \\
\hline Oiti & Chrysobalanaceae & $\begin{array}{l}\text { Licania tomentosa } \\
\text { (Benth.) Fritsch }\end{array}$ & $\mathrm{N}$ & 42 & 18,2 \\
\hline $\begin{array}{l}\text { Orelha de } \\
\text { macaco }\end{array}$ & $\begin{array}{c}\text { Fabaceae- } \\
\text { Mimosoideae }\end{array}$ & $\begin{array}{l}\text { Enterolobium contortisiliquum } \\
\text { (Vell.) Morong. }\end{array}$ & $\mathrm{N}$ & 3 & 1,3 \\
\hline $\begin{array}{l}\text { Palmeira } \\
\text { carnaúba }\end{array}$ & Arecaceae & $\begin{array}{l}\text { Copercicia prunifera } \\
\text { (Miller) H.E. Moore }\end{array}$ & $\mathrm{N}$ & 8 & 3,5 \\
\hline $\begin{array}{l}\text { Palmeira } \\
\text { imperial }\end{array}$ & Arecaceae & $\begin{array}{c}\text { Roystonea oleracea (Jacq.) } \\
\text { Cook }\end{array}$ & $E$ & 38 & 16,5 \\
\hline Pau cigarra & $\begin{array}{c}\text { Fabaceae- } \\
\text { Caeasalpinioideae }\end{array}$ & $\begin{array}{c}\text { Senna siamea } \\
\text { (LAM.) Irwim \& Barneby. }\end{array}$ & $\mathrm{E}$ & 1 & 0,4 \\
\hline Pokam & Rutaceae & Citrus reticulata Blanco & $\mathrm{E}$ & 1 & 0,4 \\
\hline Sete copas & Combretaceae & Terminalia catappa L. & $\mathrm{E}$ & 10 & 4,3 \\
\hline Sibipiruna & $\begin{array}{c}\text { Fabaceae- } \\
\text { Caeasalpinioideae }\end{array}$ & Caesalpinia peltophoroides & $\mathrm{N}$ & 2 & 0,9 \\
\hline TOTAL & & & & 231 & \\
\hline
\end{tabular}

LEGENDA: $\mathrm{N}=$ nativo; $\mathrm{E}=$ exótico

A família Arecaceae teve o maior índice, com 65 indivíduos, seguida de Chrysobalanaceae, com 42 plantas, juntamente com outras 10 famílias (FIGURA 01).

A Praça José de Anchieta, conhecida como Praça da PM, possui o maior número de exemplares, num total de 68, distribuídos em 6 famílias, 9 gêneros e 10 espécies, destacando-se a família Arecaceae, com 39 plantas, em que 25 são da espécie Roystonea oleraceae, a palmeira imperial (FIGURA 02). Alguns indivíduos vegetais $(17,64 \%)$ possuíam cupins e erva de passarinho, causando prejuízos. Foi notado que houve a realização da poda do tipo L em algumas árvores com o objetivo de retirar a fiação elétrica do 
contato com suas copas. Também a interferência das raízes na calçada foi observada, em forma de rachaduras.

A Praça Barão do Rio Branco, que recebe um número maior de pessoas por estar localizada na área central da cidade, apresentou 49 indivíduos, destacando-se a família Chrysobalanaceae, com 23 plantas da espécie
Licania tomentosa (oiti); por se tratar de uma árvore de porte alto, existem rachaduras na calçada ocasionadas pelas raízes. Apenas dois oitizeiros estavam atingindo a rede elétrica, e três Ficus benjamina (espécie conhecida como figueirinha) sofreram remoção de ramos laterais que, se não fossem eliminados, causariam um crescimento ramificado e irregular da planta.

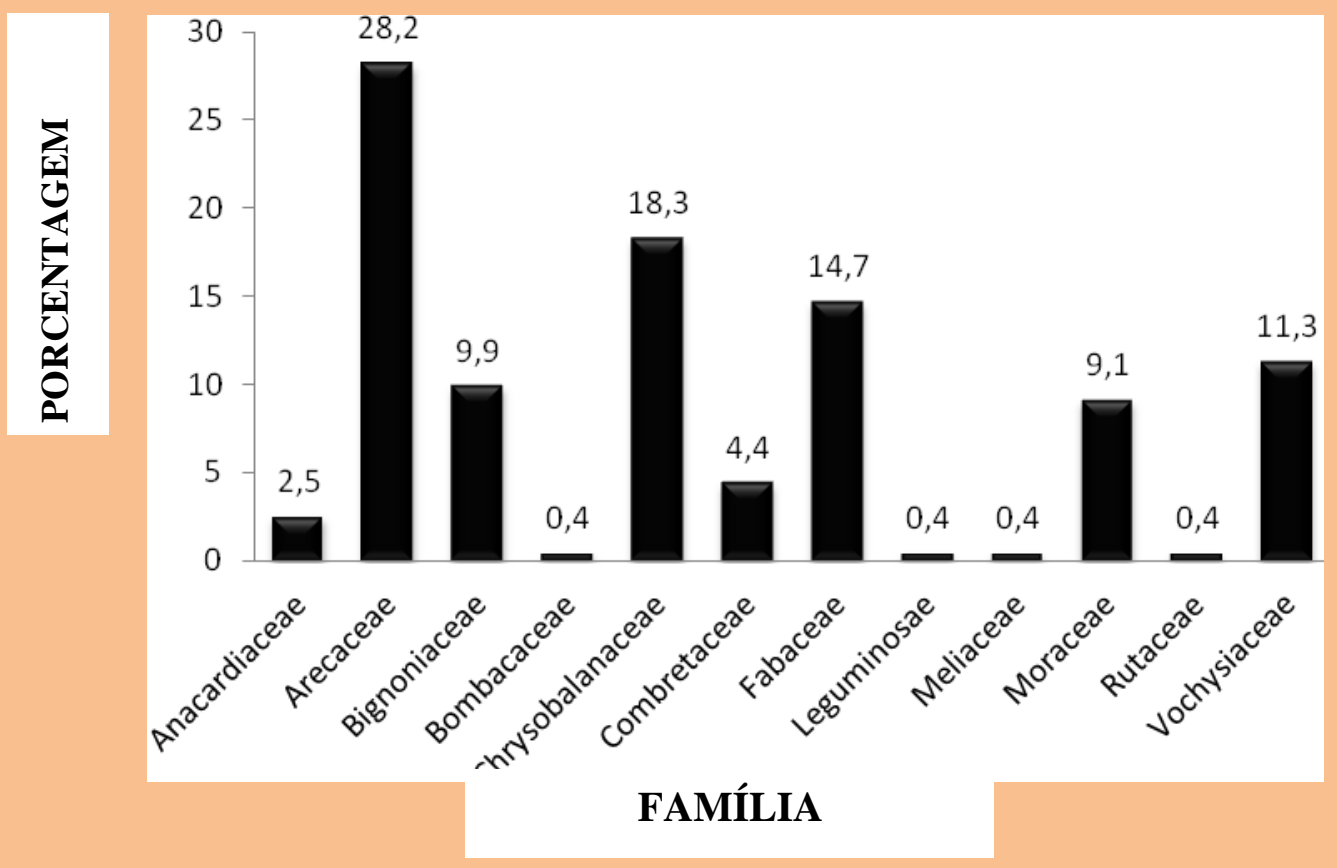

FIGURA 01: Porcentagem das famílias identificadas nas praças estudadas da cidade de Cáceres/MT, 2009. UNEMAT, 2009.

Sob a visão de Lorenzi (2003), F. benjamina não é indicada para a arborização de vias públicas, pois suas raízes são muito vigorosas, comprometendo as calçadas. Ainda neste enfoque, de acordo com Silva et. al. (2007b), esta espécie, se for plantada em calçada ou canteiro central, é sinônimo de problema no futuro, devido ao seu tipo de sistema radicular e ao seu porte, necessitando sempre de poda.

Em vários trabalhos realizados, também foi verificado que Licania tomentosa é a espécie mais utilizada na arborização de praças, como em pesquisa semelhante realizada em Uberlândia - MG por Silva et. al. (2002), em que foram identificadas 1.329 árvores pertencentes a 30 espécies, sendo Licania tomentosa, com 430 indivíduos (32\%), a mais abundante, e a segunda mais abundante Ficus benjamina, com 205 indivíduos (15 \%). Em estudos de Ibiapina et. al. (2007), em Teresina - PI, a Licania tomentosa foi a espécie mais representativa, com $61 \%$ dos 105 indivíduos amostrados.

Também vale citar o trabalho de Fava (2004) em Cuiabá - MT, no bairro Boa Esperança, em que foram amostradas 1.060 árvores. Encontraram-se 33 espécies distribuídas em 16 famílias, e Licania tomentosa foi a espécie mais abundante, com 468 indivíduos, seguida 
por Ficus benjamina, com 108 indivíduos, e Caesalpinia peltophoroides, com 96 indivíduos.

A título de comparação, observa-se também que em Itaguaí - RJ, Fícus benjamina se destaca com 50 \% do total, seguida por Terminalia catappa, com $17 \%$ (Borba, 2006); em Nova Iguaçu - RJ, Ficus benjamina é a espécie predominante, representando 21 \% (Rocha et. al., 2004), e em Curitiba, o arbusto Lagerstroemia indica constitui $19 \%$ da arborização, sendo esta a espécie de maior ocorrência (MILANO et al., 1992).

De acordo com Santamour Júnior (2002), a maior diversidade de espécies de árvores no ambiente urbano se faz necessária justamente para garantir o máximo de proteção contra pragas e doenças; o autor recomenda não exceder mais que $10 \%$ da mesma espécie, 20 \% de algum gênero e 20 \% de uma família botânica. Isso não aconteceu com os números de indivíduos de espécies e famílias, pois excederam a recomendação, com 28,2\% e $18,2 \%$, respectivamente.

Neste cenário, Gajardoni (1995) alerta sobre a importância da diversidade de espécies e que se dê preferência às árvores nativas. Em sua concepção, diversificar significa que cada espécie deve representar, no máximo, 10 \% do total de árvores da cidade.

Licania tomentosa, segunda espécie mais encontrada, não causa tanto prejuízo como Ficus benjamina, mas em geral o sistema radicular é aparente e às vezes compromete a calçada; já sua copa é densa, é uma árvore perenifólia e proporciona ótima sombra. De acordo com Lorenzi (2009), Ficus benjamina, por apresentar uma boa sombra, é ideal para plantios em praças, jardins, ruas e avenidas. Mas em considerações de Oliveira (1997), Ficus benjamina é uma árvore de grande porte e, portanto, não é ideal para o plantio em calçadas, principalmente sob rede de energia.

Na Praça Duque de Caxias, foram encontrados 59 indivíduos, pertencentes a 7 famílias, 12 gêneros e 14 espécies. Também o oitizeiro se destacou em número de indivíduos, como na Praça Barão do Rio Branco, e também estavam provocando rachaduras nas calçadas.

Na Praça Benjamim Constant, conhecida como Cavalhada, foram encontradas 10 famílias, 13 gêneros e 13 espécies, sendo 26 o maior número de indivíduos de uma mesma espécie, Vochysia divergens.

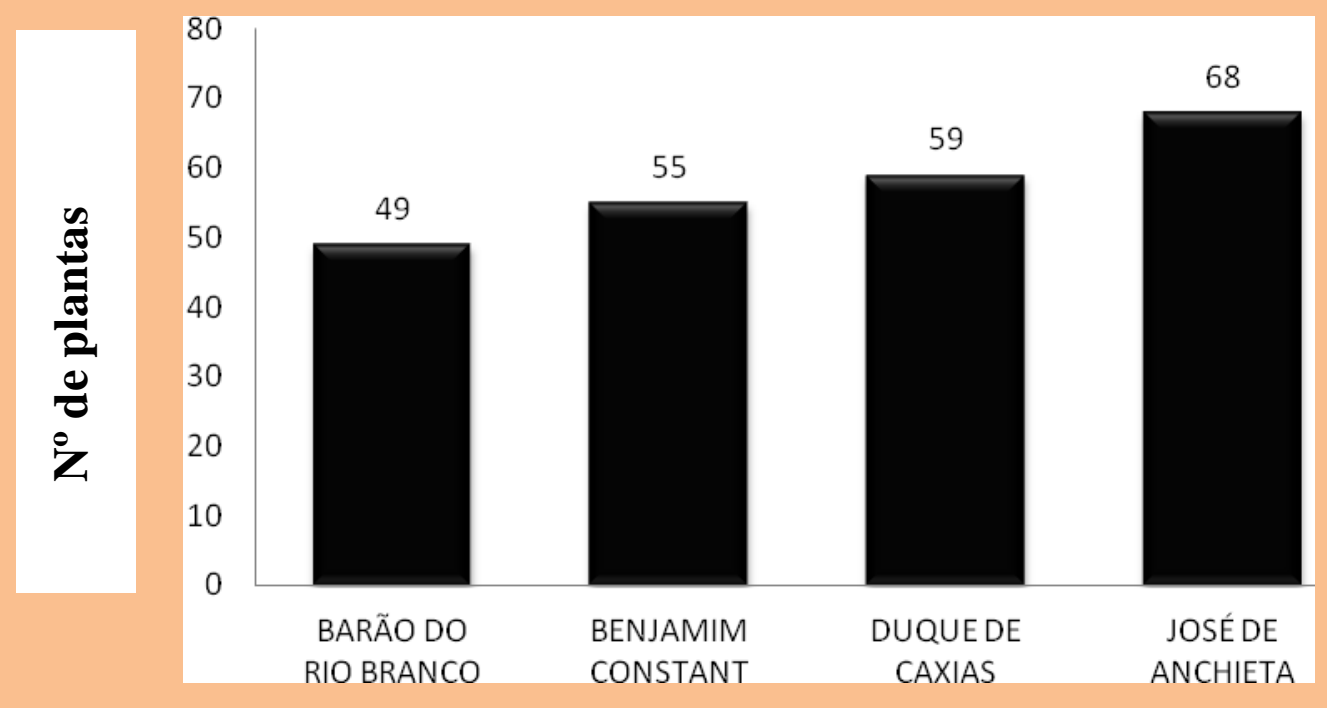

PRAÇAS

FIGURA 02: Número de individuos encontrados nas praças da região central de Cáceres/MT, 2009. UNEMAT, 2009. 
Verificou-se que a éspecie Licania tomentosa está presente em todas as praças estudadas, com 42 indivíduos, representando 18,2\%, seguida de Roystonea oleracea, com 16,5\% (FIGURA 03).

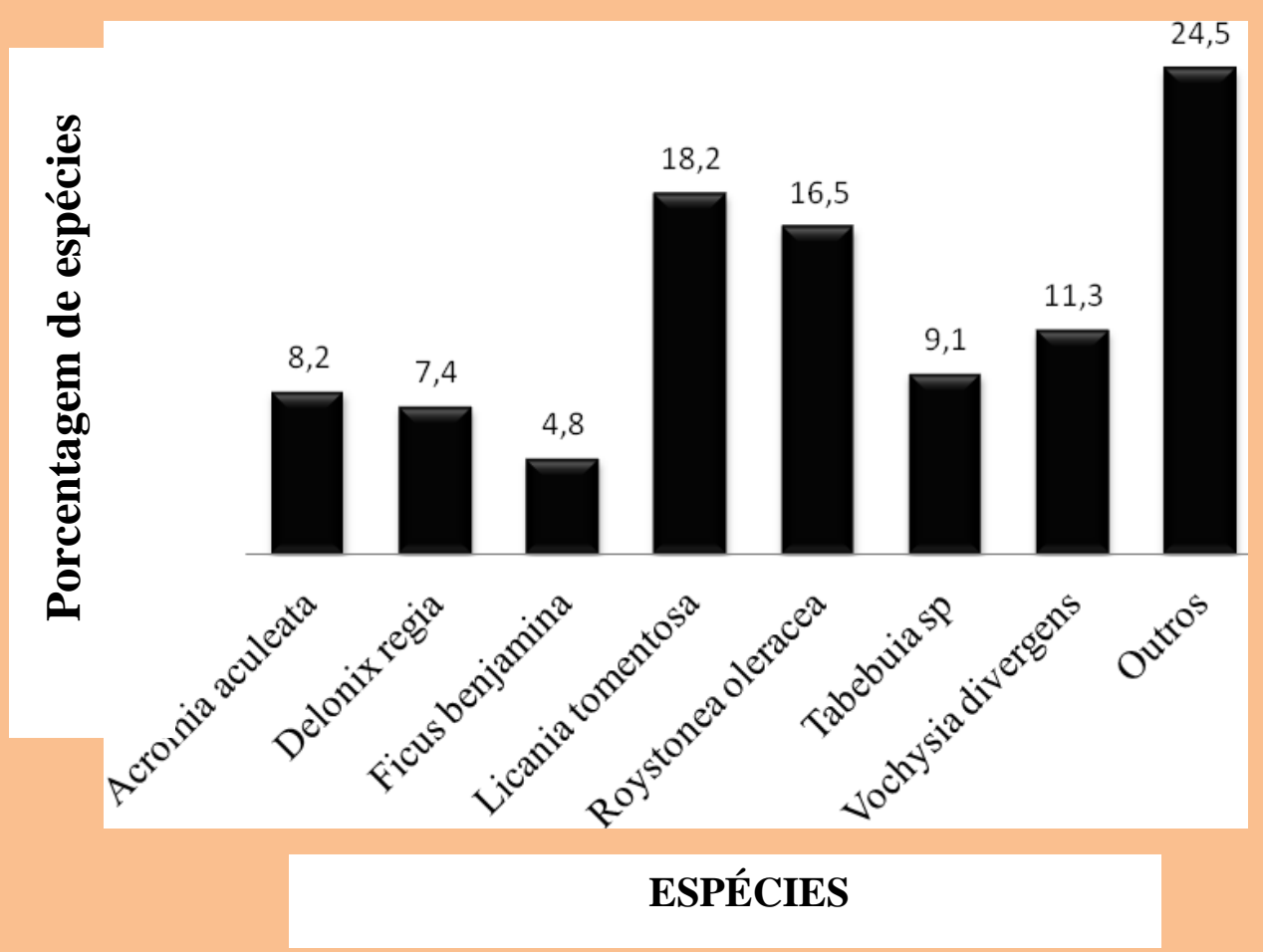

FIGURA 03: Espécies com maior percentual de presença nas quatro praças de Cáceres/MT, 2009. UNEMAT, 2009.

Em estudo realizado por Moura \& Santos (2009), que teve como objetivo realizar um levantamento qualiquantitativo de árvores e arbustos nos bairros Centro e Centro Norte, Várzea Grande - MT foi encontrada uma população de 352 árvores, na qual 33,81\% era da espécie Licania tomentosa, mostrando a preferência de árvores que ofereçam sombra, como é o caso da espécie.

Com relação à origem das árvores encontradas, das 231 estudadas, 134 são nativas e 97 são exóticas. SILVA (2007), estudando os impactos nas edificações decorrentes da arborização urbana em seis praças da cidade de Cáceres - MT, encontrou os resultados de 68\% de espécies nativas e 32\% de exóticas, chegando a valores próximos dos encontrados no presente estudo (FIGURA 04).
Resultado diferente foi encontrado no trabalho de Fava (2004) em Cuiabá - MT, correspondendo a 45,5\% de espécies nativas e 54,5 \% de exóticas. 


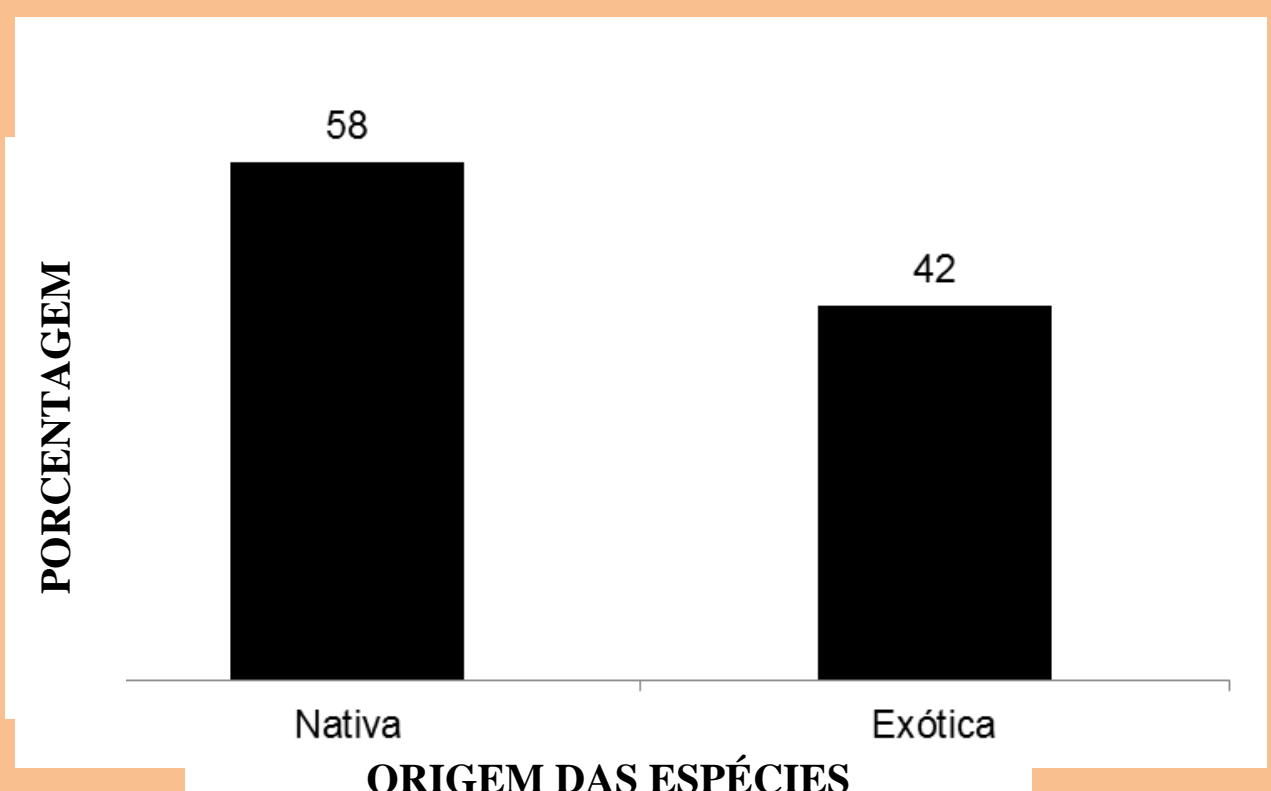

FIGURA 04: Espécies nativas e exóticas encontradas nas praças da cidade de Cáceres/MT, 2009. UNEMAT, 2009.

\section{CONCLUSÕES}

Nas quatro praças analisadas do município de Cáceres, foram encontrados ao todo 231 indivíduos, distribuídos em 25 diferentes espécies, 22 gêneros e 12 famílias botânicas.

Porém apesar do grande número de indivíduos, pode-se concluir através dos resultados obtidos que existe uma relativa uniformidade na arborização desses locais, visto que apenas 5 espécies perfazem 63,3\% da arborização total. É recomendável que seja feito o plantio de novas espécies, preferencialmente nativas para evitar a uniformidade.
A família Arecaceaea apresentou o maior número com 65 árvores, representando 28,2\% do total de plantas analisadas.

Das espécies identificadas a Licania tomentosa destacou-se por estar presente em todas as praças e a Roystonea oleracea foi a segunda mais utilizada.

O local com maior número de árvores foi a Praça José de Anchieta.

\section{REFERÊNCIAS}

BORBA, N. S. L. Levantamento da arborização viária do Centro da cidade de Itaguaí, RJ. Seropédica. Universidade Federal Rural do Rio de Janeiro, 2006. p. 48. (Monografia de Graduação)

CEMIG. Manual de arborização. Belo Horizonte, 2001.

FAVA, C. L. F. Inventário quali-quantitativo da arborização urbana do bairro Boa Esperança - Cuiabá, MT. Monografia de especialização. Lavras - MG: Universidade Federal de Lavras - UFLA, 2004. 50 p. 
GAJARDONI, A. Nossas cidades poderiam ser jardins exuberantes. Revista Globo Ciência. p. 21-27, 1995.

INSTITUTO BRASILEIRO DE GEOGRAFIA E ESTATÍSTICA (IBGE). Estimativas das populações residentes, em $\mathbf{1}^{\circ}$ de julho de 2009, segundo os municípios. 2009. 124p. Disponível em: <http://www.ibge.gov.br/home/estatistica/populacao/estimativa2009/POP2009_DOU.pdf>. Acesso em: 22 out. 2009.

IBIAPINA, A. V.; FERNANDES, D.; CARVALHO, D. C.; OLIVEIRA, E.; SILVA, M. C. A. M.; GUIMARÃES, V. S. Evolução da hidrometria no Brasil. Agência Nacional de Energía Elétrica (ANEEL), 2007. Disponível em: <http://www.mma.gov.br/port/srh/acervo/publica/doc/oestado/texto/12 1-138.html>. Acesso em: 14 ago. 2009.

LORENZI, H. Árvores brasileiras: manual de identificação e cultivo de plantas arbóreas nativas do Brasil. 3. ed. Nova Odessa: Instituto Plantarum, 2009. 384p.

LORENZI, H.; SOUZA, H. M. de; TORRES, M. A. V.; BACHER, L. B. Árvores Exóticas no Brasil: madeiras, ornamentais e aromáticas. 1.ed. Nova Odessa: Platarum, 2003. 352p.

MELO, E. F. R. Q; ROMANINI, A. Praça Ernesto Tochetto: importância da sua preservação histórica e aspectos de sua arborização. Revista da Sociedade Brasileira de arborização Urbana. Piracicaba, v. 3, n. 1, p. 54-72, 2008.

MILANO, M. S.; NUNES, M. L.; SANTOS, L. A.; SARNOWSKI FILHO, O.; ROBAYO, J. A. M. Aspectos qualiquantitativos da arborização de ruas de Curitiba (1991). In: CONGRESSO BRASILEIRO DE ARBORIZAÇÃO URBANA, 1, 1992, Vitória. Anais... Vitória: Sociedade Brasileira de Arborização Urbana, 1992. p.199- 210.

MOURA, T. A.; SANTOS, V. L. V. Levantamento quali-quantitativo de espécies arbóreas e arbustivas na arborização viária urbana os bairros centro e centro norte, várzea grande, mato grosso, brasil. Revista da sociedade brasileira de arborização urbana. v. 1, n. 1, p. 07-11, 2009.

OLIVEIRA, M. O. Arborização urbana: planejamento urbano, arborização urbana; planejamento da arborização; manutenção da arborização. Viçosa: CPT, 1997. 1 fita de vídeo. (53 min) VHS, son, color.

PIVETTA, K. F. L.; SILVA FILHO, D. F. Arborização urbana. Boletim acadêmico UNESP/FCAV/FUNEP Jaboticabal, SP, 2002. p.74.

ROCHA, R. T.; LELES, P. S. S.; NETO, S. N. O. Arborização de vias públicas em Nova Iguaçu - RJ: o caso dos bairros Rancho Novo e Centro. Revista Árvore. Viçosa. v. 28. n. 4. p. 559-607, 2004.

SANTAMOUR JÚNIOR, F. S. Trees for urban plantig: diversity uniformuty, and common sense. U.S. Nacional Abertum, Agriculture Rsearch Sevice, 2002. 
SILVA, A. G.; PAIVA, H. N.; GONÇALVES, W. Avaliando a Arborização Urbana. Viçosa: APRENDA FÁCIL, 2007a.

SILVA, A. S. S. Arborização urbana e impactos às edificações em seis praças da cidade de Cáceres-MT. Universidade do Estado de Mato Grosso, 2007. 56p. (Monografia de Graduação).

SILVA. E. M.; SILVA, A. M.; MELO, P. H.; BORGES, S. S.; LIMA, S. C. O estudo da aborização urbana do bairro Mansour, na cidade de Uberlândia -MG. IG/UFU. Caminhos de Geografia-Revista on line. v. 3, n.5, p. 73-83, fev. 2002.

SILVA, L. M.; MOCCELLIN, R.; WEISSHEIMER, D. I.; ZBORALSKI, A. R.; FONSECA, L.; RODIGHIERO, D. A. Inventário e sugestões para arborização em via pública de Pato Branco/PR. Revista da Sociedade Brasileira de Arborização Urbana, Piracicaba, v. 2, n. 1, p.101-108, 2007b. 